Internat. J. Math. \& Math. Sci.

Vol. 23, No. 10 (2000) 717-722

S0161171200002763

(c) Hindawi Publishing Corp.

\title{
THE LIFTING PROPERTY FOR CLASSES OF MAPPINGS
}

\author{
JANUSZ J. CHARATONIK
}

(Received 2 April 1999)

\begin{abstract}
The lifting property of continua for classes of mappings is defined. It is shown that the property is preserved under the inverse limit operation. The results, when applied to the class of confluent mappings, exhibit conditions under which the induced mapping between hyperspaces is confluent. This generalizes previous results in this topic.
\end{abstract}

Keywords and phrases. Arc approximation property, confluent mapping, continuum, hyperspace, induced mapping, inverse limit.

2000 Mathematics Subject Classification. Primary 54B20, 54E40.

1. Introduction. For a metric continuum $X$ we denote by $C(X)$ the hyperspace of all nonempty subcontinua of $X$. Given a mapping $f: X \rightarrow Y$ between continua $X$ and $Y$, we let $C(f): C(X) \rightarrow C(Y)$ to denote the corresponding induced mapping. Given a class $2 \mathfrak{b}$ of mappings between continua, it is well known that if a mapping $f$ is in $2 \mathfrak{b}$, then the induced mapping $C(f)$ can be, but in general need not be, in 20. Continua $Y$ for which the implication holds for all continua $X$ are named to have the $2 \mathfrak{b}$-lifting property. For example, all continua have the monotone-lifting property as well as the $O M$-lifting property, see [15, (1.212.2), page 204] and [11, Theorem 5.2, page 244], and compare [11, Theorem 3.2, page 241], while this is not the case for open-lifting property, see [11, Theorem 4.3, page 243, and example, page 244], and compare [4, Sections 4 and 5].

The class of confluent mappings comprises all classes mentioned above. Thus confluent-lifting property is of a special interest. The property was already studied in several papers. It is known that confluence of $f$ does not imply confluence of $C(f)$ (see [10, Example 5.1]; compare also [7, Example 4.1, page 131, and Example 4.24, page 143]). However, the implication is true if the range space $Y$ is a locally connected continuum (see [10, Thoerem 4.4] and [9, Theorem 2.5, page 3]) or even under a weaker assumption that the hyperspace $C(Y)$ has the arc approximation property, [7, Theorem 4.4, page 133, and Corollary 4.5, page 134]. Another result in this direction says that the implication holds provided $Y$ is the inverse limit of a sequence of locally connected continua with confluent bonding mappings, [12, Theorem 2.7, page 775].

A further progress is obtained in the present paper. The result is a common generalization of all results quoted above. Namely it is shown that the same phenomena as above for confluent mappings hold for other classes of mappings, that carried out a general statement on preserving some mapping properties of continua under the inverse limit operation. Namely, given a class 20 of mappings, a concept of a continuum having the $2 \mathfrak{b}$-lifting property has being introduced and conditions have been found 
for the class $2 \mathfrak{W}$ under which this property is preserved by the inverse limit operation. These conditions are satisfied in a particular case when the hyperspaces $C\left(Y_{n}\right)$ have the arc approximation property. Further, applying the obtained results to the class $2 \mathfrak{W}$ of confluent mappings we get some more general theorems on the confluent-lifting property. Finally, corollaries are obtained for inverse limits of some dendroids (with confluent bonding mappings).

2. Preliminaries. All considered spaces are assumed to be metric. A continuum means a compact connected metric space. A mapping means a continuous function. Given a continuum $X$ with a metric $d$, we let $2^{X}$ to denote the hyperspace of all nonempty closed subsets of $X$ equipped with the Hausdorff metric, and we denote by $C(X)$ the hyperspace of all subcontinua of $X$, i.e., of all connected elements of $2^{X}$. The reader is referred to [13] and [15] for needed information on hyperspaces.

We use terminology of [8] for inverse sequences (the set $\mathbb{N}$ of all positive integers is the index set) of spaces and of mappings. Given an inverse sequence $(X, \phi)=$ $\left\{X_{n}, \phi_{n}^{m}: m, n \in \mathbb{N}\right\}$ with factor spaces $X_{n}$ and bonding mappings $\phi_{n}^{m}: X_{m} \rightarrow X_{n}$ for every $n \leq m$ (if $n=m$, then $\phi_{n}^{n}$ is assumed to be the identity), we denote by $X_{\infty}=\varliminf_{\{}\left\{X_{n}, \phi_{n}^{m}\right\}$ its inverse limit, and by $\phi_{n}: X_{\infty} \rightarrow X_{n}$ the projections. By a mapping $\Gamma$ from the inverse sequence $(X, \phi)$ to the inverse sequence $(Y, \psi)$ we mean a sequence of mappings $\gamma_{n}: X_{n} \rightarrow Y_{n}$ such that $\gamma_{n} \circ \phi_{n}^{n+1}=\psi_{n}^{n+1} \circ \gamma_{n+1}$. The inverse limit of $\Gamma$ is a mapping $\gamma_{\infty}: X_{\infty} \rightarrow Y_{\infty}$ such that if $x=\left(x_{1}, x_{2}, x_{3}, \ldots\right) \in X_{\infty}$ then $\gamma_{\infty}(x)=\left(\gamma_{1}\left(x_{1}\right), \gamma_{2}\left(x_{2}\right), \gamma_{3}\left(x_{3}\right), \ldots\right)$. See [8, Theorem 3.13, page 218] for continuity of $\gamma_{\infty}$.

A class $2 \mathfrak{W}$ of mappings between spaces is said to have the composition property provided that for every two mappings $f: X \rightarrow Y$ and $g: Y \rightarrow Z$ belonging to $2 \mathfrak{W}$ their composition $g \circ f$ belongs to $2 \mathfrak{W}$ (see [14, Chapter 5, Part A, page 29]). A class $2 \mathfrak{W}$ of mappings between spaces is said to be neat provided that it contains all homeomorphisms, and it has the composition property.

A class $2 \mathfrak{W}$ of mappings between spaces is said to have:

- the inverse limit property provided that for every two inverse sequences $(X, \phi)$ and $(Y, \psi)$ if the mappings $\gamma_{n}: X_{n} \rightarrow Y_{n}$ are in $2 \mathfrak{W}$ for all $n \in \mathbb{N}$, then the inverse limit mapping $\gamma_{\infty}$ is in $2 \mathfrak{W}$;

- the inverse limit projection property provided that for each inverse sequence $(X, \phi)$ if the bonding mappings $\phi_{n}^{m}$ are in $2 k$ for all $n, m \in \mathbb{N}$ with $n \leq m$, then all the projections $\phi_{n}$ are in 20 , too.

The following result is known (see [3, Proposition 2.6] and compare [5, Theorem 3.3]).

THEOREM 2.1. For a neat class $2 \mathrm{~b}$ of mappings between compact spaces the inverse limit property implies the inverse limit projection property.

3. Lifting property. Let a class 20 of mappings between continua be neat. We say that a continuum $Y$ has the 2h-lifting property provided that for each continuum $X$ and each surjective mapping $f: X \rightarrow Y$ if $f$ is in 20 then the induced mapping $C(f)$ : $C(X) \rightarrow C(Y)$ is in 20 too.

The following known result (see [16, Theorem 1.1, page 707] and [15, (1.169), 
page 171]) is needed to prove the main theorem of the paper. To simplify notation we omit the subscript $\infty$ in the symbol of the inverse limit space.

Proposition 3.1. Let $Y=\varliminf_{\{}\left\{Y_{n}, \psi_{n}^{n+1}: n \in \mathbb{N}\right\}$ be the inverse limit of an inverse sequence of continua $Y_{n}$. Then the mapping

$$
h: C_{\infty}(Y)=\varliminf_{\lim }\left\{C\left(Y_{n}\right), C\left(\psi_{n}^{n+1}\right): n \in \mathbb{N}\right\} \longrightarrow C(Y)
$$

defined by $h\left(B_{1}, \ldots, B_{n}, \ldots\right)=\varliminf_{\lim }\left\{B_{n}, \psi_{n}^{n+1} \mid B_{n}: n \in \mathbb{N}\right\}$ is a homeomorphism.

THEOREM 3.2. Let a neat class $2 \mathfrak{W}$ of mappings between continua have the inverse limit property. Let $Y=\varliminf_{\{}\left\{Y_{n}, \psi_{n}^{n+1}: n \in \mathbb{N}\right\}$ be the inverse limit of an inverse sequence of continua $Y_{n}$ with bonding mappings $\psi_{n}^{n+1}: Y_{n+1} \rightarrow Y_{n}$ belonging to $2 \mathrm{~W}$. If

(*) the continua $Y_{n}$ have the $2 \mathrm{n}$-lifting property for each $n \in \mathbb{N}$, then the continuum $Y$ has the $2 \mathrm{D}$-lifting property.

Proof. The idea of this proof is taken from [12, proof of Theorem 2.7, page 775]. Let $h: C_{\infty}(Y) \rightarrow C(Y)$ be the homeomorphism defined in Proposition 3.1 and, for each $n \in \mathbb{N}$, let $\psi_{n}: Y \rightarrow Y_{n}$ be the projection. Since the bonding mappings $\psi_{n}^{n+1}$ are assumed to be in the class $2 \mathfrak{W}$ which has the inverse limit property, and thus, by Theorem 2.1, the inverse limit projection property, the projections $\psi_{n}$ are in $2 \mathfrak{W}$ for each $n \in \mathbb{N}$. Further, let a surjective mapping $f: X \rightarrow Y$ from a continuum $X$ onto $Y$ be in the class $2 \mathfrak{W}$. Since $2 \mathfrak{W}$ has the composition property, the composition $\psi_{n} \circ f: X \rightarrow Y_{n}$ is in $2 \mathfrak{W}$. By $(*)$ we infer that the induced mappings $C\left(\psi_{n}\right)$ and $C\left(\psi_{n} \circ f\right)$ are in $2 \mathfrak{W}$. On the other hand, since

$$
C\left(\psi_{n}^{n+1}\right) \circ C\left(\psi_{n+1} \circ f\right)=C\left(\psi_{n}^{n+1} \circ \psi_{n+1} \circ f\right)=C\left(\psi_{n} \circ f\right),
$$

the sequence of the mappings $\left\{C\left(\psi_{n} \circ f\right): C(X) \rightarrow C\left(Y_{n}\right): n \in \mathbb{N}\right\}$ induces the limit mapping $g: C(X) \rightarrow C_{\infty}(Y)$ defined by $g(A)=\left\{\psi_{n}(f(A)): n \in \mathbb{N}\right\} \in C_{\infty}(Y)$ for each $A \in C(X)$. Then, since $2 \mathfrak{b}$ has the inverse limit property, the limit mapping $g$ is in the class $2 \mathfrak{W}$, so is the composition $h \circ g$, the mapping $h$ being a homeomorphism. It follows from the definition of $g$ that $h \circ g=C(f)$. Thus $C(f)$ is in the class $2 \mathfrak{b}$ as needed, and the proof is complete.

A mapping $f: X \rightarrow Y$ between continua $X$ and $Y$ is said to be confluent provided that for each subcontinuum $Q$ of $Y$ each component of the inverse image $f^{-1}(Q)$ is mapped onto $Q$ under $f$. In [14] the reader can find various properties of this and related classes of mappings.

Applying the above introduced concept of the 2n-lifting property to the class 20 of confluent mappings we see that all locally connected continua have the confluentlifting property by the above quoted results of Hosokawa (see [10, Theorem 4.4] and [9, Theorem 2.5, page 3]), while the continuum constructed by Hosokawa and Kawamura in [10, Example 5.1] does not have the property.

Since the class $2 \mathfrak{b}$ of confluent mappings between continua is neat, [1, III, page 214], and has the inverse limit property, [6, Corollary 13, page 8], we obtain the following result as a consequence of Theorem 3.2. 


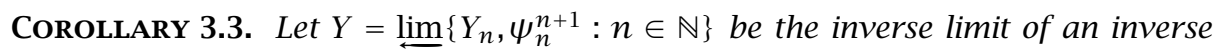
sequence of continua $Y_{n}$ with confluent bonding mappings $\psi_{n}^{n+1}: Y_{n+1} \rightarrow Y_{n}$. If $(* *)$ the continua $Y_{n}$ have the confluent-lifting property for each $n \in \mathbb{N}$, then the continuum $Y$ has the confluent-lifting property.

4. Arc approximation property. A continuum $X$ is said to be arcwise connected if every two points of $X$ can be joined by an $\operatorname{arc}$ in $X$. A continuum $X$ is said to have the arc approximation property provided that for each subcontinuum $K$ of $X$ and for each point $p \in K$ there is a sequence of arcwise connected continua $\left\{K_{n}: n \in \mathbb{N}\right\}$ in $X$ such that $p \in K_{n}$ and $K=\operatorname{Lim} K_{n}$. The reader is referred to [7] for more information on this concept.

The following result is known, see [7, Theorem 4.4, page 133].

Proposition 4.1. If the hyperspace $C(Y)$ of a continuum $Y$ has the arc approximation property, then $Y$ has the confluent-lifting property.

Applying Proposition 4.1 to each member $Y_{n}$ of the inverse sequence in Corollary 3.3 separately, we have the following theorem.

THEOREM 4.2. Let $Y=\varliminf_{n}\left\{Y_{n}, \psi_{n}^{n+1}: n \in \mathbb{N}\right\}$ be the inverse limit of an inverse sequence of continua $Y_{n}$ such that the hyperspaces $C\left(Y_{n}\right)$ have the arc approximation property, and that the bonding mappings $\psi_{n}^{n+1}: Y_{n+1} \rightarrow Y_{n}$ are confluent. If a surjective mapping $f: X \rightarrow Y$ from a continuum $X$ onto $Y$ is confluent, then the induced mapping $C(f): C(X) \rightarrow C(Y)$ is confluent, too.

If we assume that the continua $Y_{n}$ being the factor spaces of the considered inverse sequence are locally connected, then their hyperspaces $C\left(Y_{n}\right)$ are locally connected, too (see [15, (1.92), page 134]), whence it follows that they have the arc approximation property (see [9, Lemma 2.4, page 3] and compare [7, Corollary 3.7, page 115]). Thus we get the following consequence of Theorem 4.2, see [12, Theorem 2.7, page 775].

COROLlary 4.3 (Hosokawa). Let $Y=\varliminf_{\{}\left\{Y_{n}, \psi_{n}^{n+1}: n \in \mathbb{N}\right\}$ be the inverse limit of an inverse sequence of locally connected continua $Y_{n}$ such that the bonding mappings $\psi_{n}^{n+1}: Y_{n+1} \rightarrow Y_{n}$ are confluent. If a surjective mapping $f: X \rightarrow Y$ from a continuum $X$ onto $Y$ is confluent, then the induced mapping $C(f): C(X) \rightarrow C(Y)$ is confluent, too.

Recall that a Knaster's type continuum (a solenoid) means the inverse limit of arcs (of simple closed curves, respectively) with open (equivalently: with confluent, see [2, Corollaries 6.1 and 6.2, pages 228 and 229]) bonding mappings. As an application of Corollary 4.3 one gets the following result.

COROLLARY 4.4. For each confluent mapping $f$ of a continuum onto either a Knaster's type continuum or a solenoid the induced mapping $C(f)$ is confluent.

In the light of Theorem 4.2 it is interesting to know for what continua $Y$ their hyperspaces $C(Y)$ have the arc approximation property. As we already know all locally connected continua are such (see [9, Lemma 2.4, page 3] and [7, Corollary 3.7, page 115]). [7, Chapter 3] contains several results in this direction: some conditions (for arbitrary continua) are presented there which imply the property. These general 
results concern structure of continua $Y$. The reader can find their formulation and proofs in [7]. The corollaries obtained for acyclic curves are of a special interest. Some of them are reminded here. Recall that a dendroid means an arcwise connected and hereditarily unicoherent continuum. If locally connected, it is called a dendrite. A fan means a dendroid having exactly one ramification point.

STATEMENT 4.5. For the following continua $Y$ the hyperspace $C(Y)$ has the arc approximation property:

(1) locally connected continua;

(2) dendroids whose set of ramification points is contained in a dendrite; in particular, dendroids having finitely many ramification points;

(3) fans;

(4) smooth dendroids;

(5) continua $Y$ having the property of Kelley whose product with the Hilbert cube has the arc approximation property.

However, neither any structural characterization of all such continua $Y$ is known, nor interrelations between the arc approximation property of the continua and their hyperspaces are established (see [7, Questions 3.59-3.64, pages 128-129]).

Theorem 4.2 and Statement 4.5 imply the following extension of Corollary 4.3.

COROLLARY 4.6. Let $Y=\underline{\lim }\left\{Y_{n}, \psi_{n}^{n+1}: n \in \mathbb{N}\right\}$ be the inverse limit of an inverse sequence of continua $Y_{n}$ each of which belongs to one of the categories listed in Statement 4.5 such that the bonding mappings $\psi_{n}^{n+1}: Y_{n+1} \rightarrow Y_{n}$ are confluent. If a surjective mapping $f: X \rightarrow Y$ from a continuum $X$ onto $Y$ is confluent, then the induced mapping $C(f): C(X) \rightarrow C(Y)$ is confluent, too.

REMARK 4.7. One of the properties of continua which are weaker than local connectedness is the property of Kelley (see [15, (16.10) and (16.11), page 538] for the definition and the quoted result). However continua with the property of Kelley cannot be joined to those mentioned in Corollary 4.6 because an example is known (viz. [7, Example 4.7, page 135]) of continua $X$ and $Y$ both having the property of Kelley, and a confluent surjection $f: X \rightarrow Y$ such that $C(f)$ is not confluent. This shows necessity of the assumption in condition (5) of Statement 4.5 that the product of $Y$ with the Hilbert cube has the arc approximation property.

REMARK 4.8. Classes of mappings that are larger than the class of confluent mappings are ones of semi-confluent and of weakly confluent mappings (cf. [14, (vi) and (vii), page 12] for the definitions). However, there are no analogs of Theorem 4.2 and Corollary 4.6 for these two classes of mappings, as is can be seen from [7, Section 5 , page 144] for semi-confluent mappings, and from [7, Examples 6.6 and 6.8, pages 148149] for weakly confluent ones.

ACKNOwLEDGement. The author thanks Dr. W. J. Charatonik for fruitful discussion on the topic of this paper, leading to formulation of the results in a more general setting.

\section{REFERENCES}

[1] J. J. Charatonik, Confluent mappings and unicoherence of continua, Fund. Math. 56 (1964), 213-220. MR 31\#723. Zbl 134.18904. 
[2] __ Inverse limits of arcs and of simple closed curves with confluent bonding mappings, Period. Math. Hungar. 16 (1985), no. 4, 219-236. MR 87g:54074. Zbl 578.54021.

[3] _ _ Properties of elementary mappings, Acta Math. Hungar. 85 (1999), no. 1-2, 143152. CMP 1713096.

[4] J. J. Charatonik and W. J. Charatonik, Inverse limits and openness of the induced mappings, Topology Appl. to appear.

[5]_, Semi-confluent mappings, preprint.

[6] _ On projections and limit mappings of inverse systems of compact spaces, Topology Appl. 16 (1983), no. 1, 1-9. MR 85b:54016. Zbl 532.54007.

[7] W. J. Charatonik, Arc approximation property and confluence of induced mappings, Rocky Mountain J. Math. 28 (1998), no. 1, 107-154. MR 2000c:54005. Zbl 926.54024.

[8] S. Eilenberg and N. Steenrod, Foundations of Algebraic Topology, Princeton University Press, Princeton, New Jersey, 1952. MR 14,398b. Zbl 047.41402.

[9] H. Hosokawa, Induced mappings between hyperspaces, Bull. Tokyo Gakugei Univ. (4) 41 (1989), 1-6. MR 90h:54009. Zbl 692.54005.

[10] _ Induced mappings between hyperspaces. II, Bull. Tokyo Gakugei Univ. (4) 44 (1992), 1-7. MR 94a:54036. Zbl 767.54005.

[11]__ Induced mappings on hyperspaces, Tsukuba J. Math. 21 (1997), no. 1, 239-250. MR 98f:54011. Zbl 980.09485.

[12] _ _ Induced mappings on hyperspaces. II, Tsukuba J. Math. 21 (1997), no. 3, 773-783. MR 98m:54016.

[13] A. Illanes and S. B. Nadler, Jr., Hyperspaces, Marcel Dekker Inc., New York, 1999. MR 99m:54006. Zbl 990.04416.

[14] T. Maćkowiak, Continuous mappings on continua, Dissertationes Math. (Rozprawy Mat.) 158 (1979), 1-95. MR 81a:54034. Zbl 444.54021.

[15] S. B. Nadler, Jr., Hyperspaces of Sets, Marcel Dekker Inc., New York, 1978. MR 58\#18330. Zbl 432.54007.

[16] J. Segal, Hyperspaces of the inverse limit space, Proc. Amer. Math. Soc. 10 (1959), 706-709. MR 21\#7492. Zbl 093.36403.

Charatonik: Mathematical InStitute, University of WroceaW, PL.GRUNWALDZKi 2/4, 50-384, WROCŁAW, POLAND

Current address: INSTITUTO DE MATHEMÁTICAS, UNAM, CiRCUITO EXTERIOR, CIUDAD UNIVERSITARIA, 04510 MÉXICO, D. F., MÉXICO

E-mail address: jjc@ahera.math.uni.wroc.p1, jjc@gauss.matem.unam.mx 


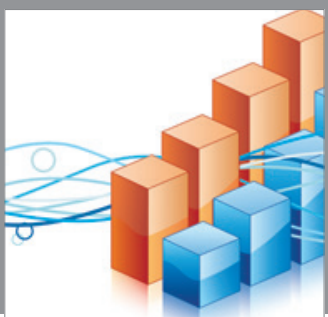

Advances in

Operations Research

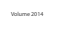

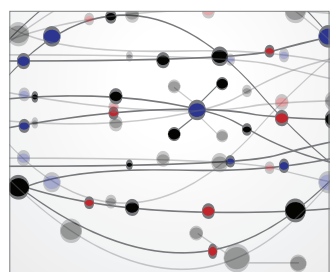

\section{The Scientific} World Journal
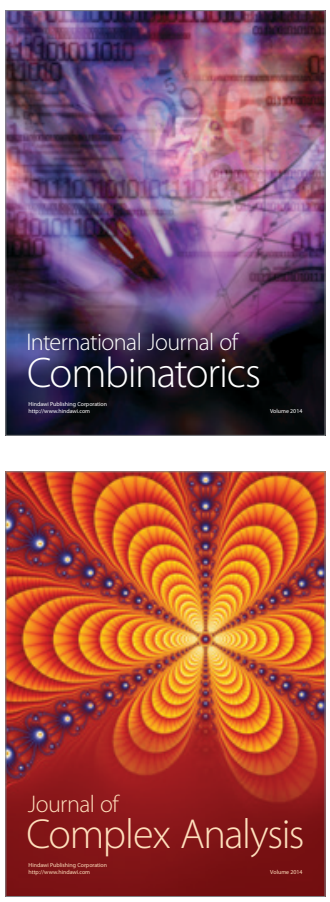

International Journal of

Mathematics and

Mathematical

Sciences
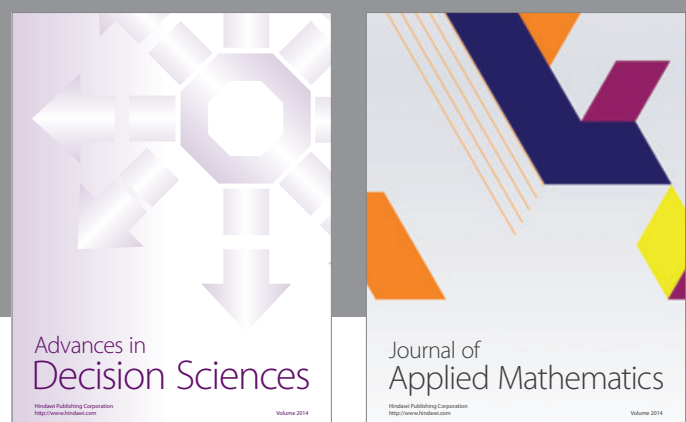

Journal of

Applied Mathematics
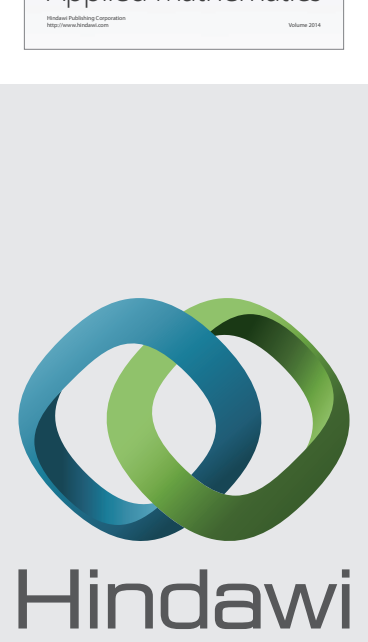

Submit your manuscripts at http://www.hindawi.com
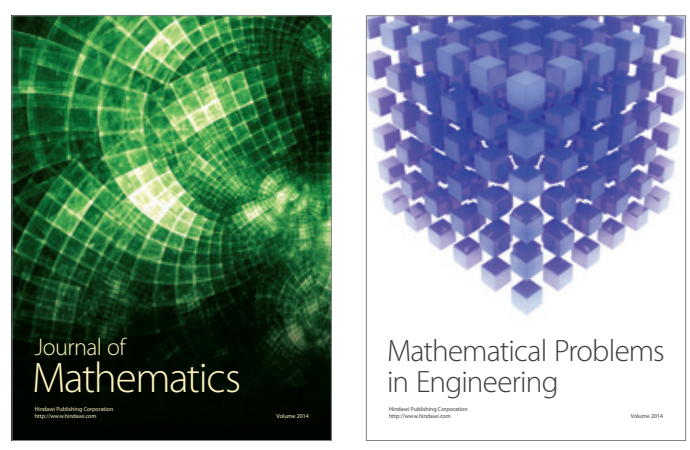

Mathematical Problems in Engineering
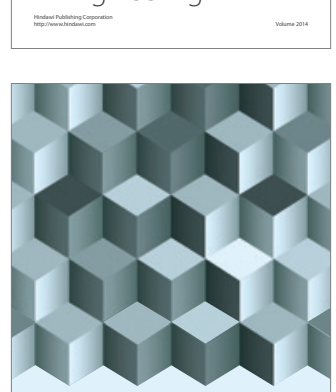

Journal of

Function Spaces
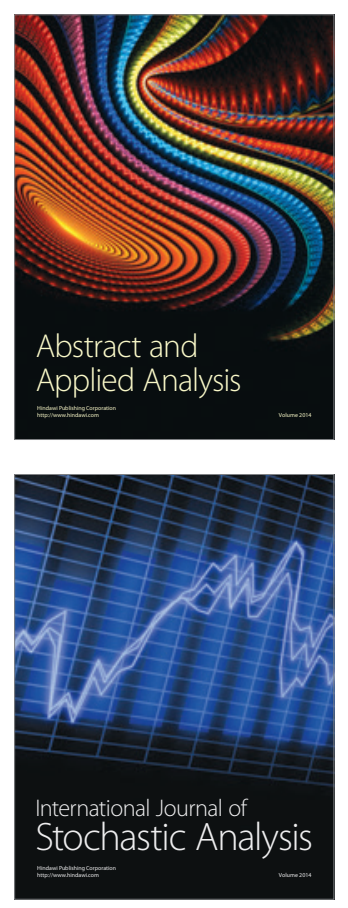

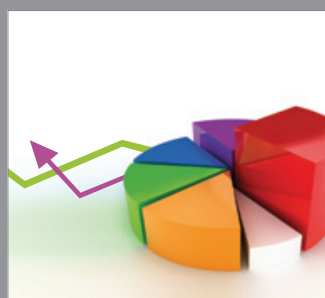

ournal of

Probability and Statistics

Promensencen
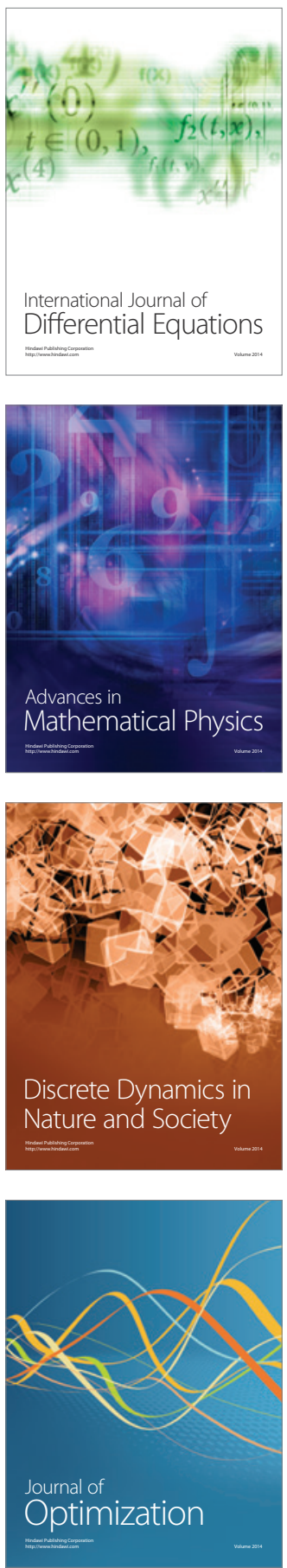